\title{
Low-Level, Global Transcription during Mitosis and Dynamic Gene Reactivation during Mitotic Exit
}

\author{
Katherine C. Palozola, ${ }^{1}$ Hong Liu, ${ }^{2}$ Dario Nicetto, ${ }^{1}$ and Kenneth S. Zaret ${ }^{1}$ \\ ${ }^{1}$ Department of Cell and Developmental Biology, Perelman School of Medicine, University of \\ Pennsylvania, Philadelphia, Pennsylvania 19104 \\ ${ }^{2}$ Department of Biochemistry and Molecular Biology, Tulane University School of Medicine, \\ New Orleans, Louisiana 70112 \\ Correspondence: zaret@upenn.edu
}

\begin{abstract}
Mitosis is thought to be a period of transcriptional silence due to the compact nature of mitotic chromosomes and the apparent exclusion of RNA Pol II and many transcription factors from mitotic chromatin. Yet accurate reactivation of a cell's specific gene expression program is needed to reestablish functional cell identity after mitosis. The majority of studies on protein regulation and localization during mitosis have relied extensively on antibodies and cross-linking-based approaches that are known to artifactually exclude proteins from mitotic chromatin. Here we show that RNA Pol II localization in mitosis is antibody- and fixation-dependent, and that direct assessment of transcription by pulse-labeling nascent RNA reveals global, low-level mitotic transcription. We also find a hierarchy of gene reactivation as the cells transition from mitosis to their interphase amplitude of gene expression. Resetting of gene transcription during mitotic exit is coincident with enhancer transcription. Our work thus shifts focus from assessing mitotic exit as a binary transcription switch to a more nuanced concert of transcription amplitude and enhancer usage. We suggest that understanding how gene expression patterns are conserved during mitosis rests upon deciphering how transcription is maintained by promoters.
\end{abstract}

During development, lineage specification and differentiation are performed by the successive expression of key transcription factors. Once a cell's functional identity has been established, it is imperative that its identity be maintained by the inheritance of the cell type-specific gene expression profile through cell division. Mitosis is the period of the cell cycle during which the recently duplicated sister chromatids are divided into two separate nuclei. At the onset of mitosis, chromosomes condense to help ensure separation of the sister chromatids. It has been thought that this condensation excludes transcriptional machinery (Martinez-Balbas et al. 1995; Prasanth et al. 2003) resulting in the termination of transcription (Prescott and Bender 1962; Parsons and Spencer 1997), and others showed a global repression of RNA synthesis by nucleotide incorporation (Prescott and Bender 1962; Konrad 1963; Johnson and Holland 1965; Parsons and Spencer 1997). However, there are caveats to each of these studies surrounding the approaches used and conclusions drawn that may have misinterpreted the transcriptional state of mitotic cells.

The carboxy-terminal domain (CTD) of Rpb1, the largest and enzymatic subunit of RNAP2, is composed of multiple, conserved heptapeptide repeats of Tyr1-Ser2Pro3-Thr4-Ser5-Pro6-Ser7. Many studies have described the role of CTD posttranslational modifications (PTMs) in the transcription regulatory cycle of recruitment, pausing, initiation, elongation, and termination (Heidemann et al. 2013). In general, phospho-serine 5 RNAP2 (Ser5-P) is found at the transcription start site and diminishes toward the $3^{\prime}$ end of the transcribed region of a gene, whereas phospho-serine 2 RNAP2 (Ser2-P) is low near the transcription start site and increases toward the 3 ' end (Komarnitsky et al. 2000; Heidemann et al. 2013). Because of these observations, and Ser2-P's dependence on the elongation factor P-TEFb (Shim et al. 2002), Ser5-P is considered the transcription initiating form of RNAP2, whereas Ser2-P is considered the elongating form of RNAP2. Notwithstanding the implications of these PTMs in transcription, both Ser2-P and Ser5-P were detected in mitotic cell extracts by western blot (WB) (Bregman et al. 1995), although it was unclear whether RNAP2 was transcriptionally engaged.

Notably, Ser2 can be phosphorylated independent of PTEFb by the atypical kinase, Brd4 (Devaiah et al. 2012), which is associated with mitotic chromatin (Dey et al. 2009). Thus, it is unclear as to whether Ser2-P is indicative of P-TEFb activity. Moreover, these studies have relied on specific CTD antibodies: the $\mathrm{H} 5$ monoclonal antibody for Ser2-P and the H14 monoclonal antibody for Ser5-P. However, H5 does not recognize a CTD that is only phosphorylated at serine 2. Rather, the epitope appears to be Ser2-P followed by Ser5-P on the same heptapeptide unit (Chapman et al. 2007). In contrast, H14 recognizes Ser5-P on one heptapeptide unit followed by Ser2-P on the next (Chapman et al. 2007). Thus, given the combinatorial potential for CTD PTMs, the transcriptional status of RNAP2 may not be reduced to a two epitope-based model.

More importantly, RNAP2 and associated proteins have been detected by mass spectrometry of mitotic chromatin (Fig. 1; Ohta et al. 2010). Also, flavopiridol-based tran-

(C) 2017 Palozola et al. This article is distributed under the terms of the Creative Commons Attribution-NonCommercial License, which permits reuse and redistribution, except for commercial purposes, provided that the original author and source are credited. 


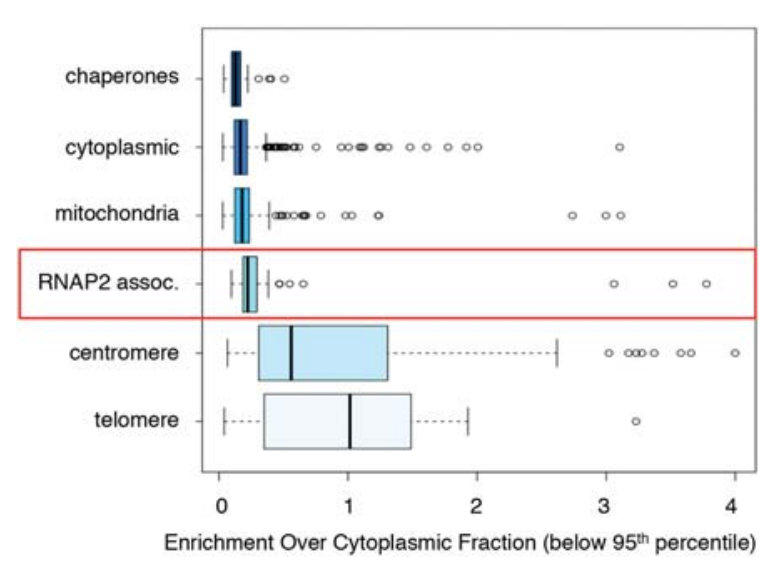

Figure 1. RNA Pol II is a structural constituent of mitotic chromatin. Analysis of mass spectrometry results from HeLa metaphase chromatin generated in Ohta et al. (2010) shows proteins enriched in chromatin compared to cytoplasmic fraction. The red box indicates proteins associated with RNAP2.

scription inhibition studies have shown that RNAP2 is associated with mitotic chromosomes (Liang et al. 2015). Though RNAP2 was not detectable in mitotic HeLa cells by chromatin immunoprecipitation followed by high-throughput sequencing (ChIP-seq), the addition of transcription elongation inhibitor resulted in paused RNAP2 peaks at several promoters in mitotic cells (Liang et al. 2015). These results suggest that low levels of transcribing RNAP2 are present, and that only when elongation is blocked and there is a backup of RNAP2 at the promoter is the signal detected. In addition, recent studies have shown that formaldehyde cross-linking artifactually removes proteins from mitotic chromatin (Lerner et al. 2016; Teves et al. 2016). Even though transcription factors were visibly excluded from mitotic chromatin by immunofluorescence (IF), fluorescently labeled fusions of the same proteins colocalized with mitotic chromatin in live cells.

Here we investigate the role that fixation- and antibodybased methods have played in establishing the traditional paradigm of transcriptional machinery exclusion from mitotic chromatin and the role of pulse-labeling transcripts in uncovering mitotic transcription. We find that there exists a global, low level of mitotic transcription that may be enhancer-independent. We also show that the genes that encode more basic cell functions are the first to increase during mitotic exit, and that enhancer activity apparently coincides with gene reactivation.

\section{RNA POL II DURING MITOSIS}

In a previous study, we used the HUH7 human hepatoma cell line to show that the pioneer factor, FoxA1, remains bound to a subset of its interphase binding sites during mitosis (Caravaca et al. 2013). Here, we use this cell line as it is amenable to efficient cell cycle synchronization and enrichment of mitotically arrested cells. Furthermore, upon release from a mitotic block, the cells efficiently reenter the cell cycle. Rather than rely on the
H5 and H14 antibodies that have traditionally been used in the literature, we performed IF for endogenous RNAP2 with an antibody raised against a synthetic RNAP2 Ser2-P peptide. We found RNAP2 Ser2-P colocalized with chromatin at every stage of mitosis (Fig. 2A-L).

Because this observation contradicts previously reported IF studies that show that RNAP2 is evicted from chromatin during mitosis, we sought to confirm the antibody's epitope specificity by preincubating the antibody with excess peptide before IF. Indeed, we found that the RNAP2 Ser2-P signal was lost in both IF (Fig. 2M-P) and WB (Fig. 2Q,R) analyses when the antibody was preincubated with the Ser2-P peptide, and that the level of Ser2-P was similar between mitotic and asynchronous cells when the antibody was not blocked. Given that the antibody we used was raised against a synthetic phosphopeptide, and not endogenous RNAP2, we needed further evidence that the epitope that we were detecting on the mitotic chromatin was in fact RNAP2 and not one of the many other phosphorylated proteins present in mitosis. To this end, we performed reciprocal immunoprecipitation (IP) followed by immunoblot (IB) analyses with the Ser2-P antibody and an antibody against the amino-terminal domain (NTD) of Rbp1, which recognizes RNAP2 independent of the CTD phospho status. In both pulldowns, the antibodies pulled down RNAP2 (Fig. 2S-V). Thus, the epitope detected on mitotic chromatin by IF is indeed a form of RNAP2.

We repeated the mitotic IF with a second Ser2-P antibody raised against the same synthetic Ser2-P peptide. This time, in agreement with previous reports, we found that RNAP2 Ser2-P was largely excluded from the mitotic chromatin (Fig. 2W,X). These results indicate that there is strong antibody dependence on RNAP2 localization in fixed cells, which reinforces the notion that formaldehyde fixation can artifactually exclude epitopes.

To circumvent the issue of fixation, we obtained a functional RNAP2-EGFP fusion construct that was previously shown to rescue temperature-sensitive RNAP2 mutants (Sugaya et al. 2000). Although the construct was lethal to the cells, live-cell imaging showed colocalization of RNAP2-EGFP with mitotic chromatin before cell death (Fig. 2Y,Z). We thus conclude that detection of RNAP2 in mitotic chromatin is both antibody dependent and that cases of exclusion are likely due to fixation artifacts, as previously reported for transcription factors (Lerner et al. 2016; Teves et al. 2016). Importantly, the presence of an active form of RNAP2 in mitosis has been reported at the centromere (Liu et al. 2015), but our observation agrees with various hints throughout the literature that it is also present at low levels throughout mitotic chromatin.

\section{MITOTIC TRANSCRIPTION}

Based on our above observation that RNAP2 can be detected on mitotic chromatin, we next sought to directly quantify RNA synthesis during mitosis. Unlike cross-linking-based approaches for detection of RNAP2, which 


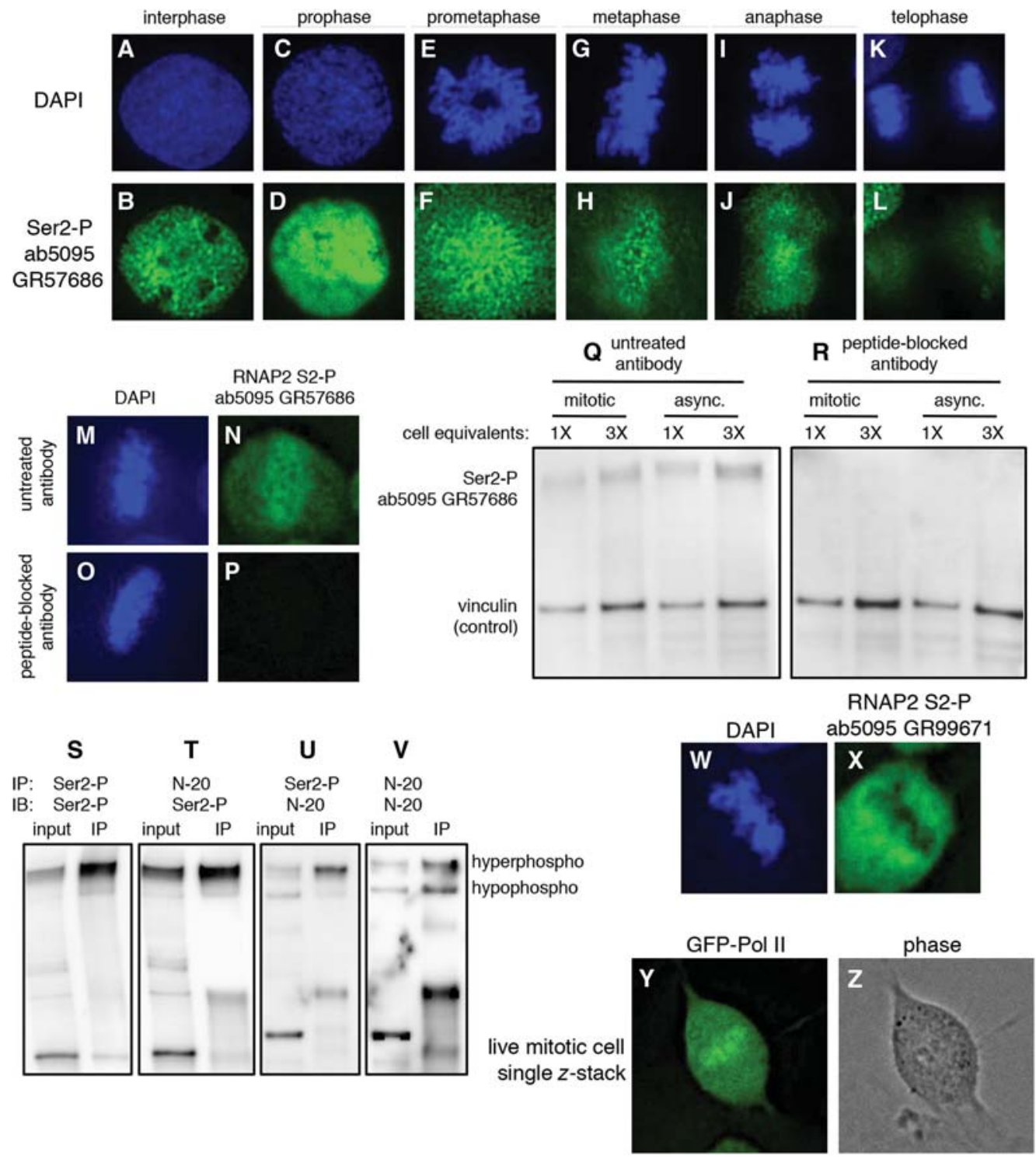

Figure 2. RNA Pol II Ser2-P colocalizes with mitotic chromatin. HUH7 cells stained with DAPI or Ser2-P in $(A, B)$ interphase, $(C, D)$ prophase, $(E, F)$ prometaphase, $(G, H)$ metaphase, $(I, J)$ anaphase, and $(K, L)$ telophase. Metaphase HUH7 cells stained with $(M)$ DAPI and $(N)$ Ser2-P, or $(O)$ DAPI and $(P)$ Ser2-P after preincubation with Ser2-P peptide. Mitotic and asynchronous HUH7 whole-cell lysate blotted with $(Q)$ Ser2-P or $(R)$ Ser2-P after preincubation with Ser2-P peptide; reciprocal immunoprecipitation (IP)-immunoblot (IB) of asynchronous HUH7 cells; $(S)$ IP with Ser2-P and blot for Ser2-P; $(T)$ IP with amino-terminal domain (NTD) and blot for Ser2-P. (U) IP with Ser2-P and blot for NTD. $(V)$ IP with NTD and blot for NTD; Ser2-P antibody, ab5095 GR57686; NTD antibody, N-20. Metaphase HUH7 cells stained with $(W)$ DAPI and $(X)$ Ser2-P; the overlay indicates Ser2-P is enriched around the periphery; Ser2-P antibody, ab5095 GR99671. ( $Y$ ) HUH7 cell transiently overexpressing GFP-RNAP2 with $(Z)$ corresponding bright field image.

indirectly assess the transcriptional state of mitotic chromatin in fixed cells, metabolic labeling of nascent transcripts with labeled nucleotide is a direct assessment of RNA synthesis in live, mitotic cells. Previously, HeLa cell metaphase spreads were incubated with FITC-UTP to allow incorporation of the FITC-UTP into any RNA currently synthesized (Liu et al. 2015). The spreads were then fixed and imaged. To aid in the analysis, the original published images were masked such that only the FITC signal that colocalized with centromeres was visible (Liu et al. 2015). Importantly, faint FITC signal was present outside of centromeres, along metaphase chromosome arms, once the mask was removed (Fig. 3A-D). Furthermore, the detected RNA synthesis was significantly reduced when the cells were treated with the transcriptional inhibitor $\alpha$ amanitin (Fig. 3E). From these results we conclude that global, low levels of transcription occur throughout mitotic chromosomes, not just at the centromeres.

The FITC-UTP study (Liu et al. 2015) also found that centromeric transcription is dependent on Bub1. The Bub1 kinase phosphorylates H2A T120 at the centromere for the recruitment of Sgo and RNAP2 to H2A T120-P 


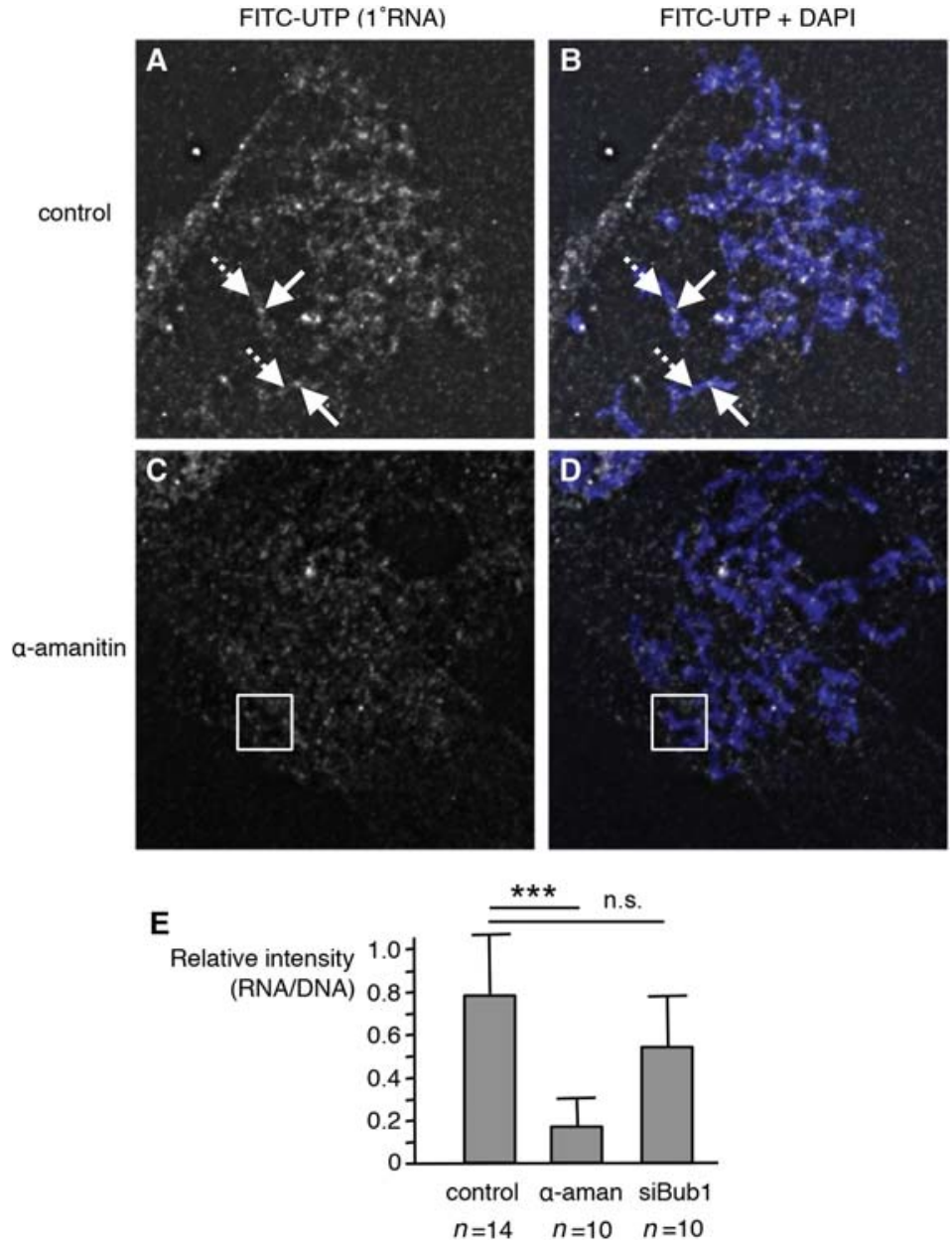

Figure 3. Transcription on metaphase chromosome arms is independent of Bub1. HeLa metaphase spreads labeled with FITC-UTP without $(A, B)$ and with $(C, D) \alpha$-amanitin treatment. Solid arrows, FITC at centromere; dashed arrows, FITC on chromosome arms; boxes, no FITC detected above background. (E) Quantification of FITC-UTP signal over chromosome arms, with and without $\alpha$-amanitin or Bub1 siRNA treatment. $n$, chromosome arms per treatment group, $P<0.001$ (two-tailed $t$-test). nucleosomes (Liu et al. 2015). When Bub1 activity is diminished by knockdown or enzymatic inhibition, RNAP2 is no longer recruited to the centromere, preventing the transcription of centromeric repeats (Liu et al. 2015). Because the transcription of these repeats is necessary for Sgo recruitment, Sgo is no longer recruited to protect the centromere, causing the cells to fail to segregate properly (Liu et al. 2015). Furthermore, ectopic expression of H2A T120-P was not sufficient to recruit RNAP2 to metaphase chromosome arms (Liu et al. 2015). We find that RNAP2 transcription along metaphase chromosome arms is Bub1-independent (Fig. 3E). Thus, mitotic transcription is occurring distinct from that at the centromere.

To assess the profile of global mitotic transcription, we developed EU-RNA-seq as a way to capture and sequence nascent RNAs in vivo (Yokoyama et al. 2016; Palozola et al. 2017). Mitotic and asynchronous cells were pulselabeled with the cell permeable uridine analog, 5'-ethynyluridine (EU) for $40 \mathrm{~min}$. The total RNA from each sample was harvested and a click reaction was performed to allow the conjugation of biotin azide to the ethynyl group of EU (Jao and Salic 2008) on any EU-RNAs present in the total RNA harvested. Once the click reaction was complete, we added custom, biotin-RNA spike-in controls to both mitotic and asynchronous samples (Palozola et al. 2017), and then streptavidin-coated magnetic beads were used to isolate biotinylated RNAs from unlabeled RNAs in each sample. We were then able to generate cDNA libraries directly off the magnetic beads for sequencing.

After global normalization between the asynchronous and mitotic samples, based on the relative spike-in sequence amplification in each replicate, there were approximately 28,000 transcripts expressed in the asynchronous population (Palozola et al. 2017). Of these, 8074 were reproducibly expressed among all three mitotic replicates, accounting for $28 \%$ of the genes expressed in an asynchronous population. However, the transcriptome was expressed much lower in mitotic than in asynchronous cells, with a fivefold mean decrement in transcript levels (Palozola et al. 2017). It is important to note that this low-level transcription is not purely the result of the $\sim 3 \%$ contaminating interphase cells in the mitotic population. If the entirety of the mitotic signal was due to an interphase population, then we would expect the relative rank of genes within this population to be identical to that of the asynchronous population. However, a pairwise analysis of 
the Spearman rank correlation coefficient of all mitotic and asynchronous replicates indicated that the two populations were distinct (Palozola et al. 2017). From these studies and other controls (Palozola et al. 2017) we conclude that mitosis is not a period of transcriptional silence, as has long been considered the case.

\section{HIERARCHICAL REACTIVATION DURING MITOTIC EXIT}

Previous studies from our laboratory (Caravaca et al. 2013) and others (Kadauke et al. 2012) measured reactivation of individual genes during mitotic exit by real time quantitative polymerase chain reaction (RT-qPCR) with primers targeting adjacent introns and exons, thus assessing primary transcripts. In doing so, it was evident that not all genes are reactivated synchronously and with the same amplitude. However, these studies were limited to a handful of genes. We thus sought to determine the hierarchy with which the transcriptome returned to interphase levels during mitotic exit, on a genome-wide level. We again used EU-RNA-seq in mitotic cells and as cells exited mitosis (Palozola et al. 2017). Intact HUH7 cells were pulsed-labeled with EU for 40 min during nocodazoleinduced mitotic arrest $\left(0^{\prime}\right)$ and at $40^{\prime}, 80^{\prime}, 105^{\prime}, 165^{\prime}$, and $300^{\prime}$ after the nocodazole was washed out and the cells were allowed to resume cycling.

To assess the various dynamics of transcription reactivation, we first removed all genes that are not expressed in asynchronous HUH7 cells. We then performed automated fuzzy c-means clustering (Schwammle and Jensen 2010) for each time point. Clusters were organized from those with genes most highly expressed at $40^{\prime}$ to those with genes most highly expressed in the asynchronous population of cells (Fig. 4). Four main patterns emerged: (1) genes that are highest at $40^{\prime}$ and then continue to decrease (Fig. 4A-D), (2) genes that are highest at $80^{\prime}$ and then continue to decrease (Fig. 4E,G,H), (3) those that increase from $40^{\prime}$ until $105^{\prime}$ or $165^{\prime}$ and then decrease (Fig. 4I-K), and (4) those that continuously increase at each time point after 40' (Fig. 4M,N). Thus, as indicated by a small number of genes in previous studies (Kadauke et al. 2012; Caravaca et al. 2013), the amplitude of the interphase

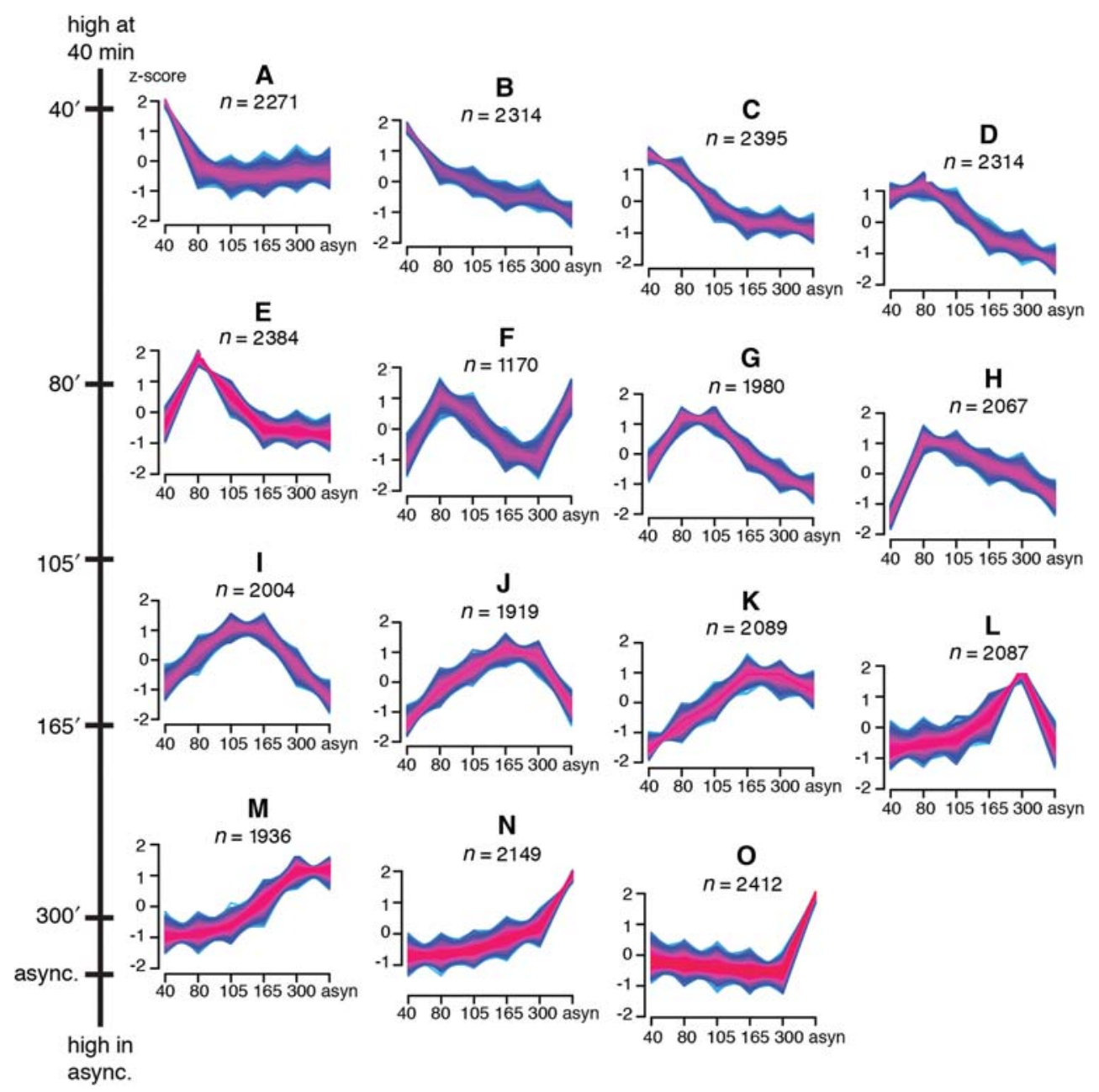

Figure 4. Dynamic patterns of reactivation during mitotic exit. Cluster analysis of the dynamic transition in expression amplitude during mitotic exit. Clusters are ordered from those highly expressed at $40^{\prime}$, to genes highly expressed in asynchronous (asyn). 
transcriptome is not achieved synchronously during mitotic exit.

We hypothesized that the first genes to increase expression during mitotic exit in our hepatoma cell line could be enriched for liver specificity. To test this, we performed Gene Ontology (GO) enrichment analysis on early- and late-activated clusters. We were surprised to find that genes highest at $40^{\prime}-80^{\prime}$ (Fig. 5A-E) are those involved in basic cellular functions such as transport and signal transduction. Conversely, genes that are highest at later time points (Fig. 5L-N) are enriched for genes involved in more cell type-specific functions like liver metabolism. However, because EU-RNA-seq measures the rate of tran- scription by EU incorporation, and shorter transcripts may undergo more rounds of transcription than longer transcripts in a given time point, we sought to determine whether gene length biased the functional gene categories at each time point. To do so, we compared the enriched $\mathrm{GO}$ categories of transcripts that first increase $\geq 1.5$-fold over mitosis at $40^{\prime}$ or $80^{\prime}$ when quantifying the FPKM (fragments per kilobase million) over the entire transcript (Fig. 5P) or the first $10 \mathrm{~kb}$ of transcripts that are $\geq 10 \mathrm{~kb}$ in length (Fig. 5Q). Indeed, the enriched GO terms in both scenarios are for basic cell functions. Thus, exiting mitosis and rebuilding daughter cells is prioritized over liver-specific gene expression in the early phases of mitotic exit.

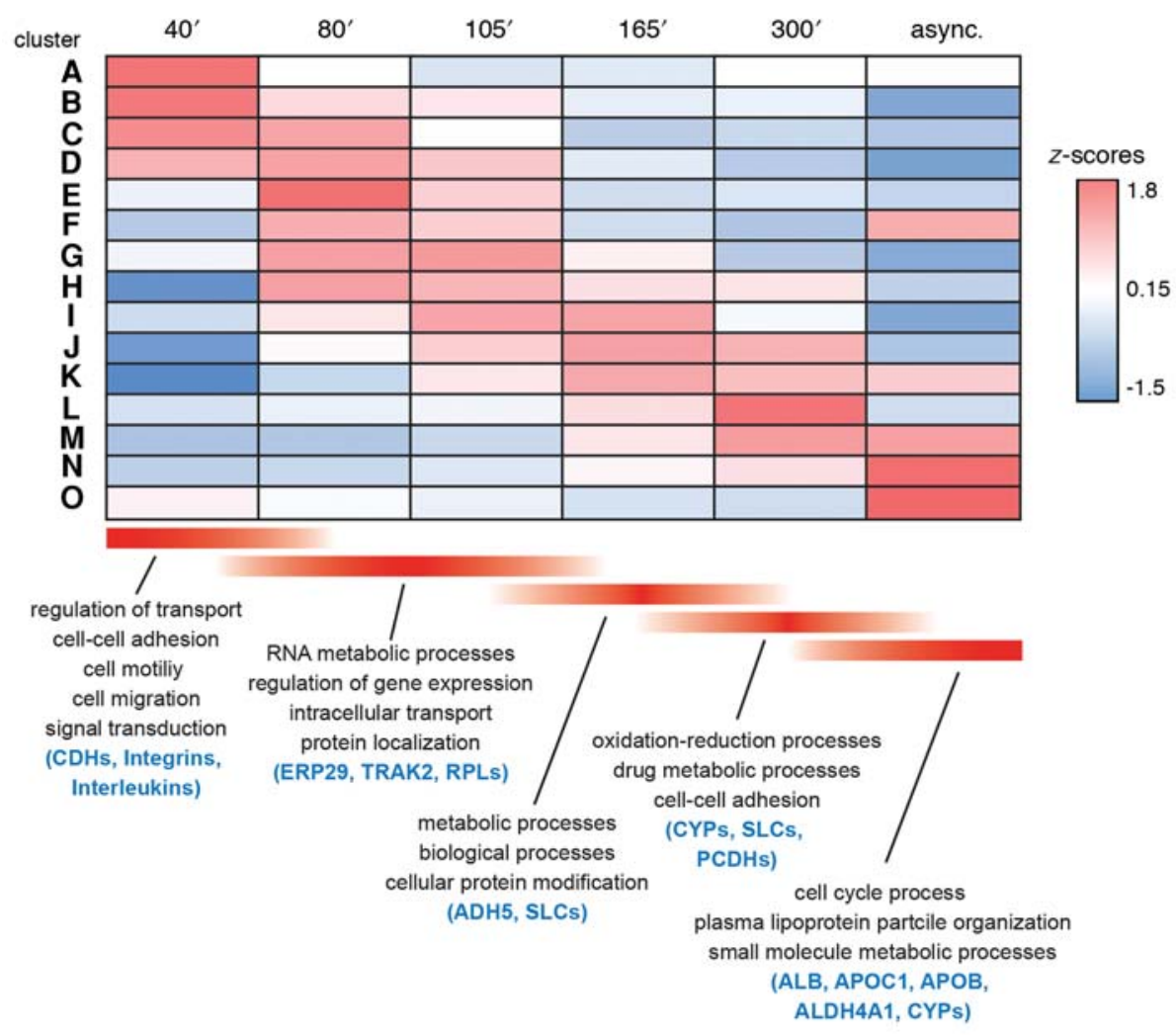

P GO terms enriched at $40^{\prime}-80^{\prime}$ for all genes

Q GO terms enriched at $40^{\prime}-80^{\prime}$ for first $10 \mathrm{~kb}$
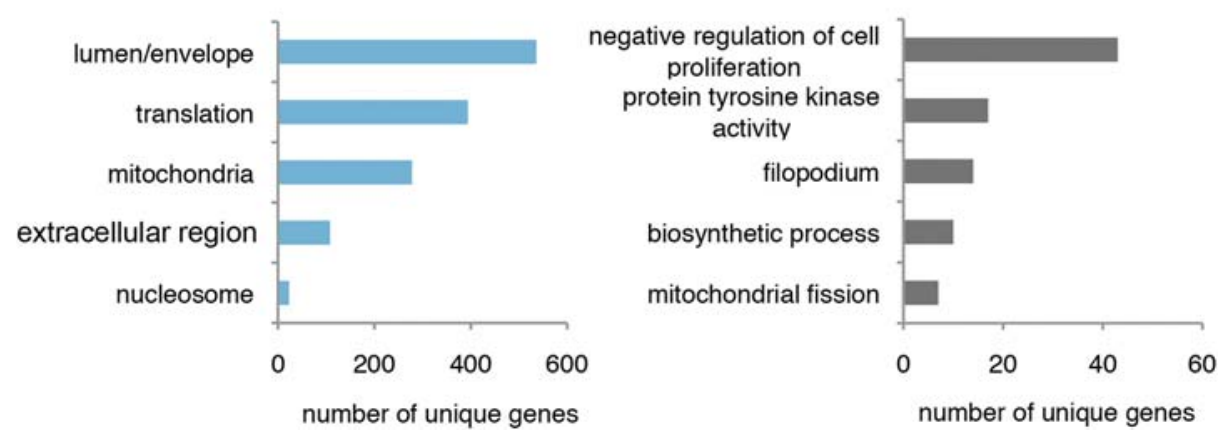

Figure 5. Basic cell functions are prioritized during mitotic exit. $(A-O)$ Heatmap of the clusters shown in Figure 4, including representative enriched GO categories. $(P)$ Representative enriched GO terms for genes that first increase $\geq 1.5$ fold over mitosis at $40^{\prime}$ or $80^{\prime}$. ( $Q$ ) Representative enriched GO terms for genes associated with the 10-kb transcript fragments that first increase $\geq 1.5$ over mitosis at $40^{\prime}$ or $80^{\prime}$. 


\section{ENHANCER USAGE}

Despite the physical condensation of mitotic chromosomes, global ATAC-seq profiles are highly similar between asynchronous and mitotic cells (Teves et al. 2016), indicating an overall retention in accessibility at the molecular level. At a finer level, it has been shown that promoter accessibility is maintained during mitosis (Martinez-Balbas et al. 1995; Hsiung et al. 2015), although enhancer accessibility is lost (Hsiung et al. 2015). Long distance interactions are also lost (Naumova et al. 2013), with enhancer-promoter loops reforming $60^{\prime} \sim 90^{\prime}$ after nocodazole washout, concordant with a spike in RNAP2 binding to enhancers and promoters (Hsiung et al. 2016).

Given the sensitivity of EU-RNA-seq, we sought to estimate the timing of enhancer usage during mitotic exit by using eRNA transcription as a surrogate for en- hancer activity. We curated all previously reported human enhancers (Leung et al. 2015) for those that both are intergenic and have a detectable signal in asynchronous HUH7 cells, and clustered them by their expression at each time point (Fig. 6). We see waves of apparent enhancer reactivation (Fig. 6A), with the largest increase occurring at $80^{\prime}$ (Fig. 6B), akin to the large burst in transcription rate increase at $80^{\prime}$ (Palozola et al. 2017), and the previously reported spike in RNAP2 binding at enhancers and reformation of enhancer-promoter contacts at 90' (Hsiung et al. 2016). However, the sensitivity of EU-RNA-seq uncovers the dynamics of enhancer usage. Interestingly, it is the eRNAs that are most lowly transcribed in asynchronous cells, which are the first to increase during mitotic exit, with the highest asynchronous eRNAs increasing later (Fig. 6C). Based on these observations, we conclude that enhancers may not be generally used during low-level mitotic transcription and are reactivated as enhancer-pro-

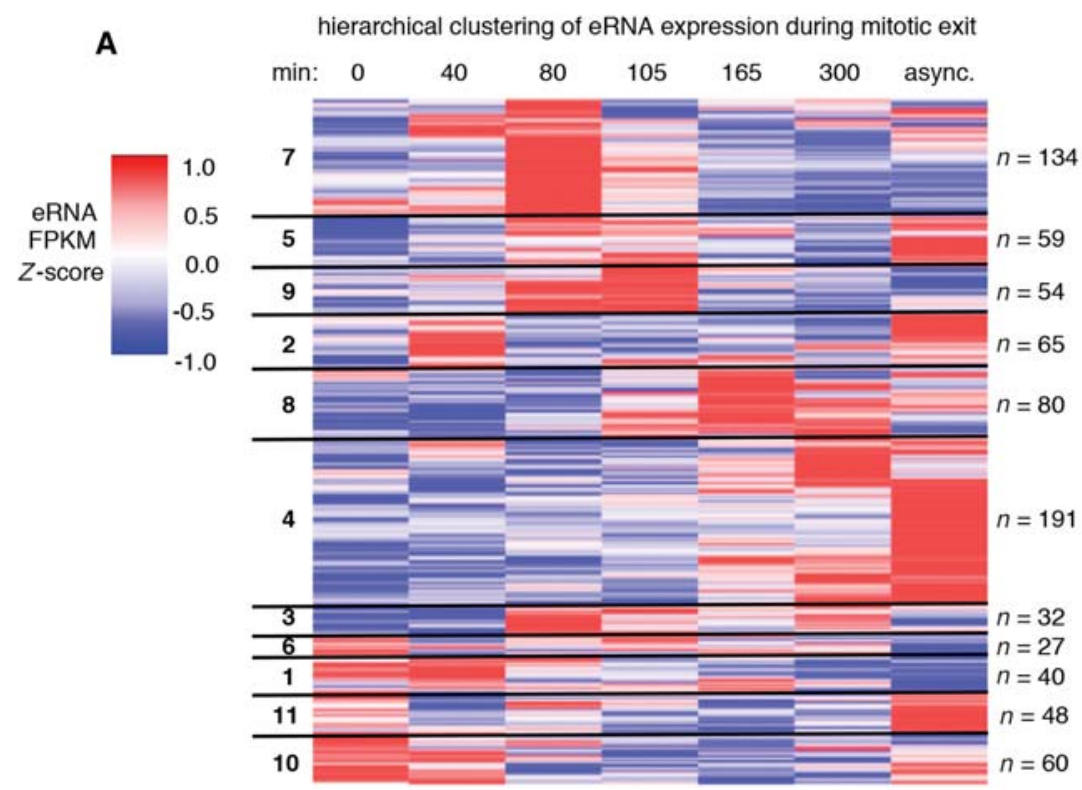

B

eRNAs increase with the largest amplitude at $80^{\prime}$
C The most highly expressed eRNAs in async. first increase at later time points

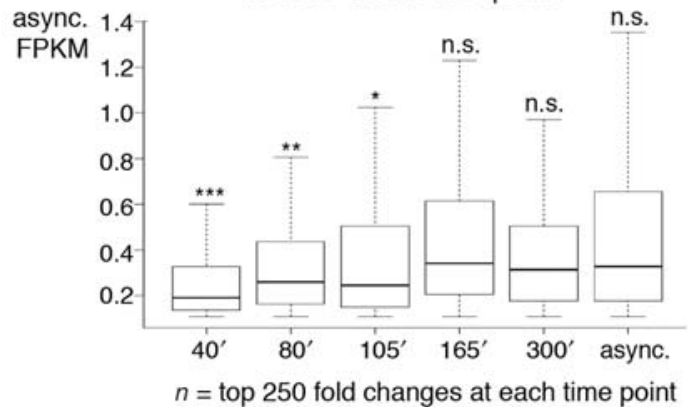

Figure 6. eRNAs are activated in waves during mitotic exit. (A) FPKM Z-score of intergenic enhancers; hierarchical cluster number on the left, number of eRNAs in each cluster on the right, $n=709$. $(B) \log _{2}$ fold increase of each eRNA over mitosis $\left(0^{\prime}\right)$ for eRNAs that first increase at each time point; $n$, top 250 eRNAs most highly expressed at that time point, Wilcoxon rank-sum test between each time point and $80^{\prime}$. (C) Asynchronous FPKM of each eRNA that first increases over mitosis $\left(0^{\prime}\right)$ for the first time at each time point; $n$, top 250 eRNAs with the highest fold change at that time point, Wilcoxon rank-sum test between each time point and asynchronous. n.s., not significant; * $P<0.05$; **, $P<0.01$; *** $P<0.001$. 

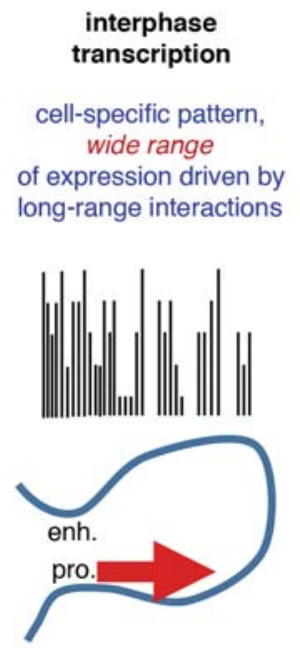

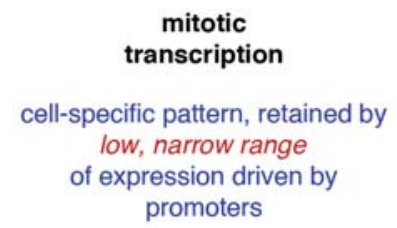

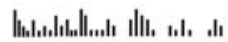

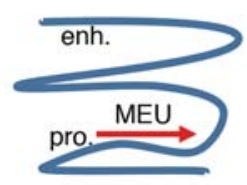

Mitotic Expression Units

\author{
transcription during \\ mitotic exit
bookmarking factors restore
amplitude
of expression

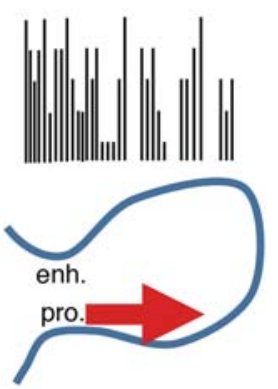

Figure 7. Enhancer usage is diminished in mitosis and is reestablished during mitotic exit. Interphase cells use enhancers to drive the cell type-specific gene expression program, whereas mitotic expression units are enhancerless and allow for continued, low-level gene expression.

moter units as long distance loops are reformed during mitotic exit.

\section{CONCLUSION}

Our increasing ability to reprogram cell identity for eventual therapeutic and analytic purposes has fostered interest in the basis of identity maintenance during mitosis. This interest was originally rooted in early observations of reduced RNA production during mitosis and then, with the increasing use of antibody-based detection methods such as IF and ChIP, the observation that RNAP2 and several transcription factors are evicted from mitotic chromatin. These latter studies have dominated the conversation and the original studies showing reduced, but not erased, transcription have been overlooked (Konrad 1963; Johnson and Holland 1965; Gariglio et al. 1974).

Here we have reexamined the mitotic transcriptional state in decades of studies. The localization of RNAP2 is both antibody- and fixation-dependent, and the complete phospho status of the CTD during mitosis remains unclear, although it does carry Ser2-P. Furthermore, global RNAP2-dependent transcription is not entirely erased. Indeed, there exists a low-level pattern of transcription throughout metaphase cells, which is distinct from that at the centromere. We also found that the interphase amplitude of gene expression is not achieved synchronously during the transition from mitosis to interphase, as gene reactivation occurs in waves, with the earliest waves including genes involved in basic cell functions. Gene reactivation appears to be congruent with enhancer usage, as eRNA activity also increases in waves during mitotic exit.

Based on these observations, and on previous reports of loss of long-range interactions (Naumova et al. 2013) and enhancer accessibility during mitosis (Hsiung et al. 2015), we propose that mitotic transcription is primarily dependent on promoters and may be largely enhancer-independent. In such a scenario, promoters drive enhancerless mitotic expression units (MEUs), much like the rudimentary transcriptional units in yeast, which lack enhancers. In this MEU model, the cell type-specific gene expression pattern is retained throughout mitosis and enhancer usage drives the reestablishment of the interphase amplitude of gene expression during mitotic exit (Fig. 7). Mutagenesis of candidate enhancers will be necessary to confirm an enhancer's putative target, and thus whether enhancer deletion affect interphase, but not mitotic, expression of the target gene. Regardless, our data, together with previous reports (Hsiung et al. 2015, 2016), indicate that the key to understanding the reestablishment of the interphase transcriptome during mitotic exit likely lies in how promoters maintain transcription of many genes during mitosis, and how enhancers are used during mitotic exit.

\section{ACKNOWLEDGMENTS}

The Zaret laboratory research described in this chapter was supported by grants from the National Institutes of Health: training grant T32GM00812 to K.C.P. and GM36477 to K.S.Z.

\section{REFERENCES}

Bregman DB, Du L, van der Zee S, Warren SL. 1995. Transcription-dependent redistribution of the large subunit of RNA polymerase II to discrete nuclear domains. J Cell Biol 129: 287298.

Caravaca JM, Donahue G, Becker JS, He X, Vinson C, Zaret KS. 2013. Bookmarking by specific and nonspecific binding of FoxA1 pioneer factor to mitotic chromosomes. Genes Dev 27: 251-260.

Chapman RD, Heidemann M, Albert TK, Mailhammer R, Flatley A, Meisterernst M, Kremmer E, Eick D. 2007. Transcribing 
RNA polymerase II is phosphorylated at CTD residue serine7. Science 318: 1780-1782.

Devaiah BN, Lewis BA, Cherman N, Hewitt MC, Albrecht BK, Robey PG, Ozato K, Sims RJ III, Singer DS. 2012. BRD4 is an atypical kinase that phosphorylates serine 2 of the RNA polymerase II carboxy-terminal domain. Proc Natl Acad Sci 109: 6927-6932.

Dey A, Nishiyama A, Karpova T, McNally J, Ozato K. 2009. Brd4 marks select genes on mitotic chromatin and directs postmitotic transcription. Mol Biol Cell 20: 4899-4909.

Gariglio P, Buss J, Green MH. 1974. Sarkosyl activation of RNA polymerase activity in mitotic mouse cells. FEBS Lett 44: 330-333.

Heidemann M, Hintermair C, Voss K, Eick D. 2013. Dynamic phosphorylation patterns of RNA polymerase II CTD during transcription. Biochim Biophys Acta 1829: 55-62.

Hsiung CC, Morrissey CS, Udugama M, Frank CL, Keller CA, Baek S, Giardine B, Crawford GE, Sung MH, Hardison RC, et al. 2015. Genome accessibility is widely preserved and locally modulated during mitosis. Genome Res 25: 213-225.

Hsiung CC, Bartman CR, Huang P, Ginart P, Stonestrom AJ, Keller CA, Face C, Jahn KS, Evans P, Sankaranarayanan L, et al. 2016. A hyperactive transcriptional state marks genome reactivation at the mitosis- $\mathrm{G}_{1}$ transition. Genes Dev 30: 1423-1439.

Jao CY, Salic A. 2008. Exploring RNA transcription and turnover in vivo by using click chemistry. Proc Natl Acad Sci 105: $15779-15784$.

Johnson TC, Holland JJ. 1965. Ribonucleic acid and protein synthesis in mitotic HeLa cells. J Cell Biol 27: 565-574.

Kadauke S, Udugama MI, Pawlicki JM, Achtman JC, Jain DP, Cheng Y, Hardison RC, Blobel GA. 2012. Tissue-specific mitotic bookmarking by hematopoietic transcription factor GATA1. Cell 150: 725-737.

Komarnitsky P, Cho EJ, Buratowski S. 2000. Different phosphorylated forms of RNA polymerase II and associated mRNA processing factors during transcription. Genes Dev 14: 2452-2460.

Konrad CG. 1963. Protein synthesis and RNA synthesis during mitosis in animal cells. J Cell Biol 19: 267-277.

Lerner J, Bagattin A, Verdeguer F, Makinistoglu MP, Garbay S, Felix T, Heidet L, Pontoglio M. 2016. Human mutations affect the epigenetic/bookmarking function of HNF1B. Nucleic Acids Res 44: 8097-8111.

Leung D, Jung I, Rajagopal N, Schmitt A, Selvaraj S, Lee AY, Yen CA, Lin S, Lin Y, Qiu Y, et al. 2015. Integrative analysis of haplotype-resolved epigenomes across human tissues. Nature 518: $350-354$.

Liang K, Woodfin AR, Slaughter BD, Unruh JR, Box AC, Rickels RA, Gao X, Haug JS, Jaspersen SL, Shilatifard A. 2015. Mitotic transcriptional activation: Clearance of actively en- gaged Pol II via transcriptional elongation control in mitosis. Mol Cell 60: 435-445.

Liu H, Qu Q, Warrington R, Rice A, Cheng N, Yu H. 2015. Mitotic transcription installs Sgol at centromeres to coordinate chromosome segregation. Mol Cell 59: 426-436.

Martinez-Balbas MA, Dey A, Rabindran SK, Ozato K, Wu C. 1995. Displacement of sequence-specific transcription factors from mitotic chromatin. Cell 83: 29-38.

Naumova N, Imakaev M, Fudenberg G, Zhan Y, Lajoie BR, Mirny LA, Dekker J. 2013. Organization of the mitotic chromosome. Science 342: 948-953.

Ohta S, Bukowski-Wills JC, Sanchez-Pulido L, Alves Fde L, Wood L, Chen ZA, Platani M, Fischer L, Hudson DF, Ponting CP, et al. 2010. The protein composition of mitotic chromosomes determined using multiclassifier combinatorial proteomics. Cell 142: 810-821.

Palozola KC, Donahue G, Liu H, Grant GR, Becker JS, Cote A, Yu H, Raj A, Zaret KS. 2017. Mitotic transcription and waves of gene reactivation during mitotic exit. Science 358: 119-122.

Parsons GG, Spencer CA. 1997. Mitotic repression of RNA polymerase II transcription is accompanied by release of transcription elongation complexes. Mol Cell Biol 17: 57915802.

Prasanth KV, Sacco-Bubulya PA, Prasanth SG, Spector DL. 2003. Sequential entry of components of the gene expression machinery into daughter nuclei. Mol Biol Cell 14: 10431057.

Prescott DM, Bender MA. 1962. Synthesis of RNA and protein during mitosis in mammalian tissue culture cells. Exp Cell Res 26: $260-268$.

Schwammle V, Jensen ON. 2010. A simple and fast method to determine the parameters for fuzzy c-means cluster analysis. Bioinformatics 26: 2841-2848.

Shim EY, Walker AK, Shi Y, Blackwell TK. 2002. CDK-9/ cyclin $\mathrm{T}(\mathrm{P}-\mathrm{TEFb})$ is required in two postinitiation pathways for transcription in the C. elegans embryo. Genes Dev 16: 2135-2146.

Sugaya K, Vigneron M, Cook PR. 2000. Mammalian cell lines expressing functional RNA polymerase II tagged with the green fluorescent protein. J Cell Sci 113(Pt 15): 2679-2683.

Teves SS, An L, Hansen AS, Xie L, Darzacq X, Tjian R. 2016. A dynamic mode of mitotic bookmarking by transcription factors. Elife 5: e22280.

Yokoyama Y, Zhu H, Lee JH, Kossenkov AV, Wu SY, Wickramasinghe JM, Yin X, Palozola KC, Gardini A, Showe LC, et al. 2016. BET Inhibitors suppress ALDH activity by targeting ALDH1A1 super-enhancer in ovarian cancer. Cancer Res 76: 6320-6330. 


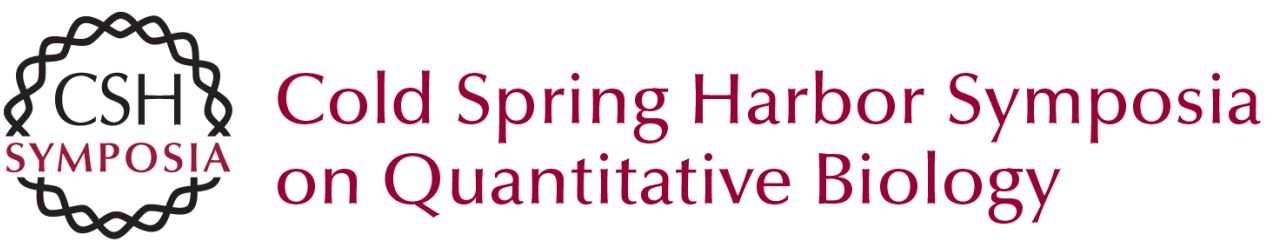

\section{Low-Level, Global Transcription during Mitosis and Dynamic Gene Reactivation during Mitotic Exit}

Katherine C. Palozola, Hong Liu, Dario Nicetto, et al.

Cold Spring Harb Symp Quant Biol 2017 82: 197-205 originally published online January 18, 2018

Access the most recent version at doi:10.1101/sqb.2017.82.034280

References This article cites 30 articles, 18 of which can be accessed free at: http://symposium.cshlp.org/content/82/197.full.html\#ref-list-1

Creative This article is distributed under the terms of the

Commons http://creativecommons.org/licenses/by-nc/4.0/, which permits reuse and License redistribution, except for commercial purposes, provided that the original author and source are credited.

Email Alerting Receive free email alerts when new articles cite this article - sign up in Service the box at the top right corner of the article or click here. 